\title{
RELATIVE COLLARING
}

\author{
LEONARD R. RUBIN
}

\begin{abstract}
It was proved by Morton Brown that the boundary of a manifold has a collar neighborhood. Later Robert Connelly gave a different proof of that result, and his method of proof was used by T. Benny Rushing to prove a relative collaring theorem. This paper contains a relative collaring theorem that is more general than either of the two just mentioned.
\end{abstract}

In [Co] a collaring theorem is stated that generalizes a result of Morton Brown's and provides a different proof of it. Some of this work is duplicated in [Da]. A similar result, Relative Collaring Theorem 1.7.7, is given in [Ru] where the previous collaring result is generalized in that it is made relative, but is specialized in that it deals with manifolds only. Furthermore, the proof in [Ru] is given for a pair $(N, M)$ where both $N$ and $M$ are compact, but the result need not be true in case $M$ is not closed in $N$, whether or not $N$ is compact. To correct this requires a change in the definition of local collaring; this in turn provides the framework for the general result that will be stated below.

Definition. Let $N_{0}$ be a subspace of a space $N$ and $\pi$ be a collection of subspaces of $N$. Let $\mathfrak{R}_{0}=\left\{M_{0}=M \cap N_{0} \mid M \in \mathfrak{R}\right\}$. We say $\left(N_{0}, \mathfrak{R}_{0}\right)$ is locally collared in $(N, \mathfrak{R})$ if for each $x \in N_{0}$ there is an $N_{0}$ neighborhood $U$ of $x$ and an embedding $h: U \times[0,1) \rightarrow N$ such that

(i) $h(u, 0)=u$ for all $u \in U$;

(ii) $h(U \times(0,1)) \subset N-N_{0}$;

(iii) $h(U \times[0,1))$ is a neighborhood of $x$;

(iv) $h(U \times[0,1)) \cap M=h\left(\left(M_{0} \cap U\right) \times[0,1)\right)$ for all $M \in \Re$.

We say $\left(N_{0}, \Re_{0}\right)$ has a (closed) collar $h$ in $(N, \mathfrak{N})$ if there is an (a closed) embedding $h: N_{0} \times[0,1] \rightarrow N$ such that

(i) $h(x, 0)=x$ for all $x \in N_{0}$;

(ii) $h\left(N_{0} \times[0,1)\right)$ is open in $N$;

(iii) $h\left(N_{0} \times[0,1]\right) \cap M=h\left(M_{0} \times[0,1]\right)$ for all $M \in \Re$.

If $\mathfrak{R}$ is the singleton $\{\boldsymbol{M}\}$, we just write $(N, \boldsymbol{M})$ in place of $(N, \mathfrak{R})$ and $\left(N_{0}, M_{0}\right)$ in place of $\left(N_{0}, \mathfrak{N}_{0}\right)$ in the above definitions.

We are now ready to state a quite general relative collaring theorem.

THEOREM 1. If $N$ is a paracompact Hausdorff space, $N_{0}$ is closed in $N$, and $\left(N_{0}, \mathfrak{T}_{0}\right)$ is locally collared in $(N, \mathfrak{T})$, then $\left(N_{0}, \mathfrak{T}_{0}\right)$ has a closed collar in $(N$, T).

Presented to the Society, January 26, 1975; received by the editors November 11, 1974 and, in revised form, January 21, 1975.

AMS (MOS) subject classifications (1970). Primary 54C25; Secondary 57A40.

Key words and phrases. Locally collared, closed collar. 
For convenience, we state here a result due to E. A. Michael [Mi].

LEMMA 1. Let $X$ be a paracompact Hausdorff space and $\mathcal{G}$ a collection of subsets of $X$ that contains an open cover of $X$. Suppose the following are true:

1. If $U \in \mathcal{G}$ and $W$ is open in $U$, then $W \in \mathcal{G}$.

2. If $\mathcal{H} \subset \mathcal{G}$ is a collection of open subsets of $X$ such that $\{\bar{H} \mid H \in \mathcal{H}\}$ is a pairwise disjoint, locally finite collection of subsets of $X$, then $\cup\{H \mid H \in \mathcal{H}\}$ $\in \mathcal{G}$.

3. If $U, V$ are open elements of $\mathcal{G}$, then $U \cup V \in \mathcal{G}$.

Then the entire space $X$ is in $\mathcal{G}$.

This follows from Proposition 3.3 of [Mi]. The best way to see this is to note that it follows from the proof of 3.6(a) of [Mi]. The reader should observe that our requirement 2 is equivalent to requiring $H$ be discrete. This serves to demonstrate that in paracompact spaces, in the definition of $G$-hereditary given by Michael, condition (c) could be relaxed to read, "discrete collection of open sets," instead of "disjoint collection of open sets."

The next lemma will also be useful in the sequel.

LEMMA 2. Let $A$ be a paracompact Hausdorff space and $Q$ be a neighborhood of $A \times 0$ in $A \times[0,1]$. Then there exists a closed embedding $\theta: A \times[0,1]$ $\rightarrow A \times[0,1]$ such that $\theta(A \times[0,1]) \subset$ int $Q, \theta(a, 0)=a$ for all $a \in A$, and $\theta(A \times[0,1))$ is an open subset of $A \times[0,1]$. We may choose $\theta$ so that $\theta(a \times[0,1]) \subset a \times[0,1]$ for all $a \in A$.

Proof. We may as well assume $Q$ is open and $Q \subset A \times[0,1)$. First suppose $t: A \rightarrow(0,1]$ is a map such that $(a, t(a)) \in Q$ for all $a \in A$. Define $\theta: A \times[0,1] \rightarrow A \times[0,1]$ by $\theta(a, s)=(a, s \cdot t(a))$. Then $\theta$ is an embedding. Continuity of $t$ can be used to show $\theta(A \times[0,1])$ is a closed subspace of $A \times[0,1]$ so that $\theta$ is a closed map. Similarly one shows $\theta(A \times[0,1))$ is open in $A \times[0,1]$. Hence we need only prove the existence of the map $t$.

For each $a \in A$, let $w(a)$ be the first element of $[0,1]$ such that $(a, w(a))$ $\notin Q$. We shall prove $w$ is lower semicontinuous; i.e., that $w^{-1}(d, 1]$ is open in $A$ for all $d \in[0,1]$. Suppose $y \in w^{-1}(d, 1]$ and that every neighborhood of $y$ contains a point $z$ such that $w(z) \leqq d$. Then there is a set $\left\{z_{\alpha}\right\} \subset A-y$ such that $y$ is a limit point of $\left\{z_{\alpha}\right\}$ and $w\left(z_{\alpha}\right) \leqq d$ for all $\alpha$. It is easy to show, since $[0,1]$ is compact, that $\left\{\left(z_{\alpha}, w\left(z_{\alpha}\right)\right)\right\}$ must have some limit point $(y, u)$ $\in A \times[0, d]$. Since $u<w(y),(y, u) \in Q$, implying some $\left(z_{\alpha}, w\left(z_{\alpha}\right)\right)$ belongs to $Q$, which is a contradiction. This proves $w$ is lower semicontinuous.

Using VIII, 4.3 of [Du], there is a map $t: A \rightarrow[0,1]$ such that $0<t(a)$ $<w(a)$ for all $a \in A$. This completes the proof of Lemma 2 .

Proof of TheOREM 1. An application of the regularity of $N$ to the definition of local collaring shows that for each $x \in N_{0}$ there exist open subsets $V, W$ of $N_{0}$ with $x \in V \subset \bar{V} \subset W$ and a closed embedding $h: \bar{W} \times[0,1] \rightarrow N$ such that,

(i) $h(w, 0)=w$ for all $w \in \bar{W}$;

(ii) $h(\bar{W} \times(0,1]) \subset N-N_{0}$;

(iii) $h(W \times[0,1))$ is open in $N$;

(iv) $h(\bar{W} \times[0,1]) \cap M=h\left(\left(M_{0} \cap \bar{W}\right) \times[0,1]\right)$ for all $M \in \Re$. 
Define $\mathcal{G}$ to be the set of all open sets $V$ as above; then $\mathcal{G}$ contains an open cover of $N_{0}$ which is paracompact, being a closed subspace of a paracompact space. Clearly $\mathcal{G}$ satisfies 1 of Lemma 1 . So suppose $\mathcal{H}$ is a collection of open subsets of $N_{0}$ as in 2 of Lemma 1 . Then $\{\bar{H} \mid H \in \mathcal{H}\}$ is a locally finite collection of subsets of $N$ as well as of $N_{0}$ because $N_{0}$ is closed in $N$. Paracompactness of $N$ can be used to show there is a collection of open sets $\left\{Q_{H} \mid H \in \mathcal{H}\right\}$ with $\left\{\bar{Q}_{H} \mid H \in \mathcal{H}\right\}$ a pairwise disjoint, locally finite collection and with $\bar{H} \subset Q_{H}$ for all $H$. For a given $V \in \mathcal{H}$ there is a $W$ with $V \subset \bar{V} \subset W$ and an embedding $h: \bar{W} \times[0,1] \rightarrow N$ satisfying (i)-(iv) above. Applying normality to cut down the set $W$ if necessary, we may assume $h(\bar{W} \times 0) \subset Q_{V}$. An application of Lemma 2 permits us to assume $h(\bar{W} \times[0,1]) \subset Q_{V}$. Piecing these maps $h$ together, we get an embedding from $(\cup\{\bar{W}\}) \times[0,1]$ into $N$ having the desired properties. This proves $\cup\{H \mid H \in \mathcal{H}\}$ is in $\mathcal{G}$ so that 2 is satisfied.

To show that 3 is also true one may proceed as in [Co] or as in $[\mathbf{R u}, \mathrm{pp}$. 42-43]. A collar $N_{0} \times[-1,0]$ is attached to $N$ at $N_{0}$ along $N_{0} \times 0$ to form the space $N^{+}$. Then, since we have only two open sets to deal with, say $U, V$, we do not necessarily cover the entire set $N_{0}$ as in [Ru]. In two steps, a closed embedding $g$ of $N$ into $N^{+}$is constructed such that $g$ merges the fibers emanating from a neighborhood of $\bar{U}$ into $N$ with those emanating from a neighborhood of $\bar{V}$ into the natural fibers of $N_{0} \times[-1,0]$. This is done in such a way that $g^{-1}\left(g(N) \cap\left(N_{0} \times[-1,0]\right)\right)$ contains a collar neighborhood of $\bar{U} \cup \bar{V}$ of the desired type. The details of the construction of $g$ are essentially those of the proofs found in $[\mathbf{C o}]$ and $[\mathbf{R u}]$ mentioned above, so we do not reproduce them here.

Since, by Lemma 1 , these steps imply $N_{0} \in \mathcal{G}$, the proof of Theorem 1 is complete.

Now let $(N, M)$ be an allowable manifold pair as in [Ru, p. 42]. Although similar, our definition of locally collared is different from the one used there by Rushing. Furthermore, if $\partial N \cap M \neq \partial N \cap \bar{M}$, then it is possible that $(\partial N, \partial N \cap M)$ not be locally collared in $(N, M)$ in our sense. We therefore do not see how Rushing's proof can be extended in case it is not true that $\partial N \cap M=\partial N \cap \bar{M}$.

We now state our Relative Collaring Theorem, which follows easily from Theorem 1.

THEOREM 2. Let $(N, M)$ be an allowable manifold pair. If the pair $(\partial N, \partial N \cap M)$ is locally collared in $(N, M)$, then it has a closed collar in $(N, M)$.

In case $N_{0}$ is not closed in $N$, we can still obtain corollaries to Theorem 1 that yield open collars.

COROllary 1. Suppose $N_{0}$ is a subspace of a Hausdorff space $N$ such that $N_{0}=U \cap V$ where $U$ is open in $N$ and is a paracompact space, while $V$ is a closed subspace of $N$. If $\left(N_{0}, \Re_{0}\right)$ is locally collared in $(N, \Re)$, then $\left(N_{0}, \Re_{0}\right)$ has a collar in $(N, \mathfrak{R})$.

Proof. Since $U$ is an open neighborhood of $N_{0}$ in $N$, it is easy to see that $\left(N_{0}, \mathfrak{N}_{0}^{\prime}\right)$ is locally collared in $\left(U, \mathfrak{K}^{\prime}\right)$ where $\mathfrak{K}^{\prime}=\{M \cap U \mid M \in \mathfrak{M}\}$. 
Since $N_{0}$ is closed in $U$, we can apply Theorem 1 to find a closed collar $h: N_{0} \times[0,1] \rightarrow U$. Since $h\left(N_{0} \times[0,1)\right)$ is open in $U$, it is also open in $N$.

Corollary 2. Suppose $N_{0}$ is a locally compact subspace of a hereditarily paracompact Hausdorff space $N$ (e.g., $N$ is metrizable). If $\left(N_{0}, \mathfrak{T}_{0}\right)$ is locally collared in $(N, \mathfrak{R})$, then $\left(N_{0}, \mathfrak{T}_{0}\right)$ has a collar in $(N, \mathfrak{R})$.

Proof. This follows from Corollary 1 because a locally compact subspace of a Hausdorff space is the intersection of an open subspace with a closed subspace.

Our results hold in the PL category also. One may consult [Co], [Ru] or [Da] to see why this is true.

\section{REFERENCES}

[Co] Robert Connelly, A new proof of Brown's collaring theorem, Proc. Amer. Math. Soc. 27 (1971), 180-182. MR 42 \#2490.

[Da] Jerome Dancis, Shrinking collapsible sets in topological manifolds and embedding manifolds "rel" the boundary, University of Maryland Technical Report TR 74-49, 1974, 25 pp.

[Du] James Dugundji, Topology, Allyn and Bacon, Boston, Mass., 1966. MR 33 \# 1824.

[Mi] E. A. Michael, Local properties of topological spaces, Duke Math. J. 21 (1954), 163-171. MR 15, 977. 1973.

[Ru] T. Benny Rushing, Topological embeddings, Academic Press, New York and London,

Department of Mathematics, University of OkLahoma, Norman, OKlahoma 73069 